\title{
An Enhanced Location Management Scheme for Hierarchical Mobile IPv6 Networks
}

\author{
Myung-Kyu Yi \\ Dept. of Computer Science \& Engineering Korea University, \\ 1,5-Ga, Anam-Dong, SungBuk-Gu, Seoul 136-701, South Korea \\ kainos@disys.korea.ac.kr
}

\begin{abstract}
In this paper, we propose an enhanced location management scheme for minimizing signaling costs in hierarchical Mobile IPv6 (HMIPv6) networks. If the mobile node's mobility is not local, in our proposal, the mobile node sends location update messages to correspondent nodes in the same way as Mobile IPv6 (MIPv6). After the creation of a spatial locality of the mobile node's movement, the mobile node sends location update messages to the correspondent nodes in same way as HMIPv6. Therefore, our proposal can reduce signaling costs in terms of packet transmission delays in HMIPv6 networks. Therefore, our proposal offers considerable performance advantages to MIPv6 and HMIPv6.
\end{abstract}

\section{Introduction}

Mobile IPv6 (MIPv6) provides an efficient and scalable mechanism for host mobility within the Internet [1. It allows an IPv6 node to be mobile and arbitrarily change its location on the IPv6 Internet while still maintaining existing connections. However, MIPv6 causes in a high signaling cost when it updates the location of an Mobile Node (MN) if it moves frequently 2. Thus, Hierarchical Mobile IPv6 (HMIPv6) is proposed by IETF to reduce signaling costs [2]. It uses a new MIPv6 node called the Mobility Anchor Point (MAP) to handle Mobile IP registration locally. It is well known that the performance of HMIPv6 is better than that of MIPv6 2. This is especially true when the basic assumption is that $69 \%$ of a user's mobility is local. If the user's mobility is not local, performance of HMIPv6, in terms of delays for packet delivery, is worse than that of MIPv6, due to the encapsulation processing by the MAP. Since all packets from a Correspondent Node $(\mathrm{CN})$ to an $\mathrm{MN}$ are first delivered through the MAP, it is possible that the MAP can become bottlenecked. Therefore, the load of the search and tunnelling processes increase on the MAP as the number of MNs increase in the foreign or home networks. It is a critical problem for the performance of HMIPv6 networks. To overcome these drawbacks, we will introduce an enhanced location management scheme, called AHMIPv6, for minimizing signaling costs in HMIPv6 networks. In our proposal, location registration with the Home Agent (HA) and MAP is exactly the same as that in HMIPv6. However, the $\mathrm{MN}$ sends a Binding Update (BU) message to the $\mathrm{CN}$ with either on-Link 


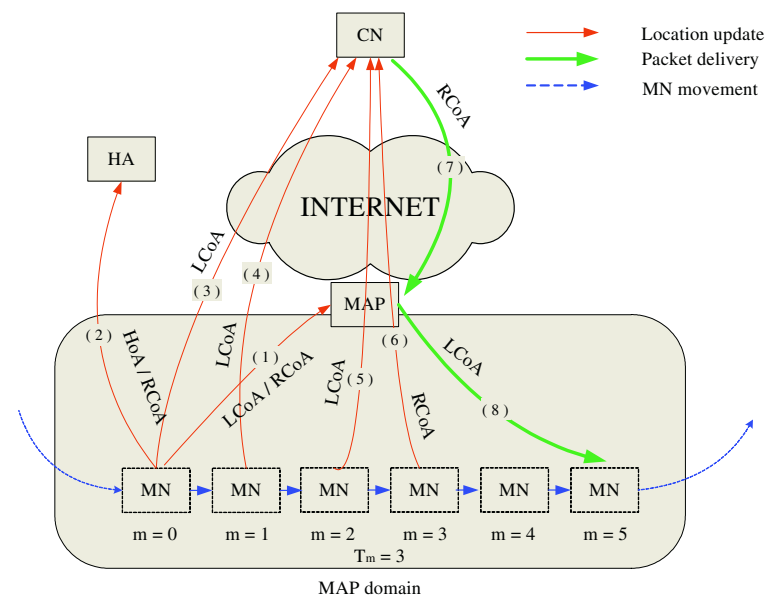

Fig. 1. The System Model for the Proposed Scheme in HMIPv6 Networks

Care-of-Address (LCoA) or Regional CoA (RCoA), based on the geographical locality properties of the MN's movements. To do so, we define $m$ and $T_{m}$ as the number of subnet crossings and the value of the movement threshold to decide whether the MN's mobility is local or not, respectively. If $m$ is less than $T_{m}$ (i.e., $m<3$ in Fig. 1 ), the MN sends a BU message to the CN with the LCoA. Otherwise, if $m$ is greater than or equal to $T_{m}$, the MN sends a BU message to the $\mathrm{CN}$ with the RCoA. Therefore, the $\mathrm{CN}$ can directly send packets to the MN without the intervention of the MAP, and before the creation of geographical locality properties of the MN's movements.

The rest of the paper is organized as follows. Section 2 describes the proposed procedures of location update and packet delivery using the enhanced mobility management scheme called AHMIPv6. Finally, our conclusions are presented in Section 3.

\section{Protocol Description}

This section describes the location update and packet delivery procedure.

\subsection{Location Update Procedure}

Similar to HMIPv6, each MN has two addresses, an RCoA and an LCoA. Each MN has a value of $m$ and $T_{m}$. While $m$ is the number of subnet crossings within the MAP domain, $T_{m}$ is the threshold value which decides whether the MN sends a BU message to the CN with the LCoA or the RCoA. Whenever an MN enters a new MAP domain, it sets the value of $m$ to zero. $T_{m}$ can be adjusted based on the user's mobility pattern and current traffic load.

Fig. 2 presents the procedure for a location update by sending the BU message. The procedures for a location update are as follows: 


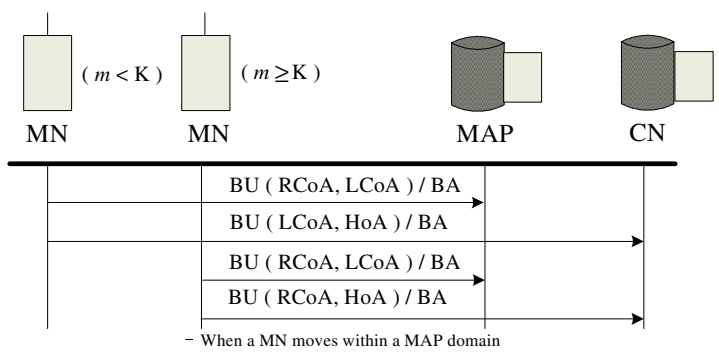

Fig. 2. The Proposed Location Update Procedure

- If an MN moves to a different MAP domain, then:

1) the MN obtains two CoAs: an LCoA and an RCoA.

2) Then, it registers with its MAP and HA by sending a BU message, and it sets the value of $m$ to zero.

- Otherwise, if an MN moves within the same MAP domain, then:

1) the MN gets a new LCoA.

2) The MN registers with its MAP by sending a BU message.

After registration with the MAP, the MN compares the value of $m$ with $T_{m}$.

Case 1. If the value of $m$ is less than $T_{m}$, then:

3-1) the MN sends a $B U_{[H o A, L C o A}[$ message to the $\mathrm{CN}$.

Case 2. Otherwise, if the value of $m$ is greater than or equal to $T_{m}$, then:

3-2) the MN sends a $B U_{[H o A, R C o A}$ message to the CN. After the sending of the $B U_{[H o A, R C o A]}$ message, the $\mathrm{MN}$ does not send any other BU messages to the $\mathrm{CN}$ before it moves out of the MAP domain.

As a result, the MN performs registration with the CNs using an $\mathrm{RCoA}$ or an LCoA, depending on its mobility pattern.

\subsection{Packet Delivery Procedure}

Fig. 3 presents the procedure for packet delivery in our proposal. In our proposal, the packet delivery procedure is exactly the same to that in MIPv6 or HMIPv6. The CN does not need to consider whether it has an RCoA or an LCoA of the $\mathrm{MN}$ in its binding cache entry. If a $\mathrm{CN}$ has an LCoA of the $\mathrm{MN}$ in its binding cache entry, it sends packets directly to an MN. Thus, the proposed scheme can achieve optimal routing, the same as MIPv6. Otherwise, if a $\mathrm{CN}$ has an $\mathrm{RCoA}$

\footnotetext{
${ }^{1}$ BU with the binding between the MN's HoA and LCoA.

${ }^{2} \mathrm{BU}$ with the binding between the MN's HoA and RCoA.
} 


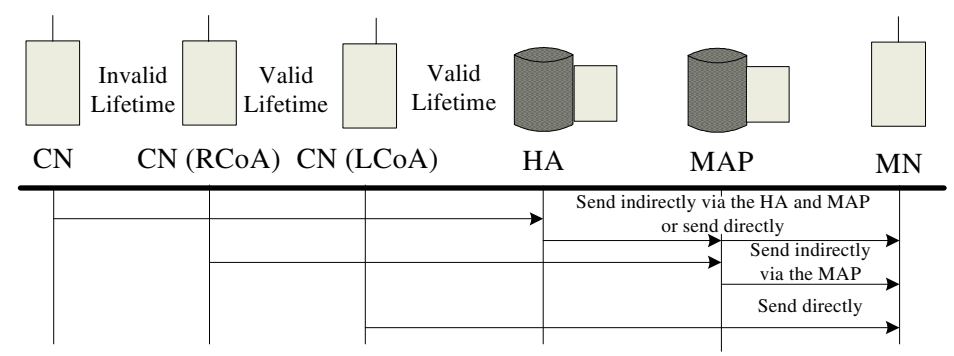

Fig. 3. The Proposed Packet Delivery Procedure

of the MN in its binding cache entry, it must send the packets to the MAP first using an RCoA. Then, the MAP encapsulates and forwards them directly to the $\mathrm{MN}$. If a $\mathrm{CN}$ has no binding cache for the $\mathrm{MN}$, it sends the packets to the home link, then the HA intercepts and tunnels them to the MN using the LCoA or RCoA.

\section{Conclusions}

In this paper, we proposed an enhanced location management scheme for minimizing signaling costs in HMIPv6 networks. In our proposal, location registration with the HA and MAP is exactly the same as that in HMIPv6. However, the $\mathrm{MN}$ sends a BU message to the $\mathrm{CN}$ with either an LCoA or an RCoA, based on the geographical locality properties of the MN's movements. To do so, we define $m$ and $T_{m}$ as the number of subnet crossings and the value of the movement threshold, to decide whether the MN's mobility is local or not, respectively. If $m$ is less than $T_{m}$, the MN sends a BU message to the CN with an LCoA. Otherwise, if $m$ is greater than or equal to $T_{m}$, the MN sends a BU message to the $\mathrm{CN}$ with an RCoA. Therefore, the CN can directly send packets to the MN without the intervention of the MAP, before the creation of geographical locality properties of the MN's movements. Therefore, our proposal achieves significant performance improvements by using the MN's selection to send a BU message to the $\mathrm{CN}$, either with an LCoA or an RCoA.

\section{References}

1. D. B. Johnson, C. E. Perkins, and J. Arkko, "Mobility support in IPv6," IETF Request for Comments 3775, June 2004.

2. H. Soliman, C. Castelluccia, K. El-Malki, and L. Bellier, "Hierarchical Mobile IPv6 mobility management (HMIPv6)", IETF Internet draft, draft-ietf-mipshop-hmipv602.txt (work in progress), June 2004. 